\title{
Integration of quantitative and qualitative descriptors for genetic diversity studies of watermelon accessions
}

\author{
Mariana Neto Rosa Lima ${ }^{1,2 *}$, Manoel Abilio de Queiróz ${ }^{2}$, Anne Emanuelle Flor da Silva Oliveira ${ }^{2}$, \\ Izaias da Silva Lima Neto ${ }^{1}$, Ronaldo Simão de Oliveira ${ }^{3}$
}

${ }^{1}$ Centro de Ciências Agrárias, Universidade Federal do Vale do São Francisco - UNIVASF, Rodovia BR 407, KM 119, Lote 543 PSNC, s/n, C1, CEP 56300-990, Petrolina-PE, Brazil

${ }^{2}$ Departamento de Tecnologias e Ciências Sociais, Universidade do Estado da Bahia - UNEB, Av. Edgard Chastinet, s/n, São Geraldo, CEP 48900-000, Juazeiro-BA, Brazil

${ }^{3}$ Departamento de Ciências Biológicas, Universidade Estadual de Feira de Santana - UEFS, Avenida Transnordestina, S/N. Novo Horizonte, CEP 44036-900 Feira de Santana-BA, Brazil

*Corresponding author: mariana.neto@univasf.edu.br

\begin{abstract}
Most of genetic diversity studies are based only on quantitative descriptors. The aim of this study was to apply different analysis strategies with quantitative and qualitative descriptors to identify which is the most suitable to integrate descriptors for genetic diversity studies of watermelon (Citrullus lanatus) germplasm using different assays. A total of 20 watermelon accessions were evaluated in the State of Rio Grande do Norte and two commercial cultivars were used as witness (control) (Crimson Sweet and Charleston Gray) in two cultivation seasons (2014/2015), using 49 descriptors, in which 26 were quantitative and 23 were qualitative. The genetic diversity of the accessions was obtained using eight analysis strategies that combined the Mahalanobis Distance, Average Standardized Euclidean Distance (SED), and Weighted Average Euclidean Distance, followed by groupings using the Tocher optimization method. A wide morphological variability was observed between and within accessions. The eight different strategies of genetic dissimilarity analyses resulted in 36 different groups. The clustering strategy, by which dissimilarity between accessions calculated using SED (quantitative descriptors) and WED (qualitative descriptors) is the most suitable for integrating quantitative and qualitative descriptors in genetic diversity studies of watermelon germplasm.
\end{abstract}

Keywords: Citrullus lanatus, multivariate analysis, dissimilarity measures, genetic diversity, landraces.

Abbreviations: ANOVA_Analysis of variance, MD_Mahalanobis Distance, SED_Average Standardized Euclidean Distance, WED_Weighted Average Euclidean Distance.

\section{Introduction}

The success of crop improvement programs depends on the existence and knowledge of the variability of the species of interest. Characterization and agronomic evaluation are essential steps in germplasm management as they comprise a detailed description of accessions regarding morphological, agronomic, molecular traits and reaction to diseases among others. Characterization is performed by applying descriptors that aid in defining, sorting and identifying the individuals of species and these descriptors might be divided into two groups: quantitative and qualitative (Cruz et al., 2011). Quantitative descriptors are real-scale, measurable characteristics such as fruit mass and soluble solids. They are generally polygenic and highly influenced by the environment; nonetheless, they are of great interest due to their economic importance (Cruz et al., 2011; Crossa and Franco, 2004). Qualitative descriptors do not have quantitative values and might be binary or multi-categorical (defined by several categories or classes), i.e., they play a role in the classification of individuals, e.g. pulp color and fruit shape (Sudré et al., 2006). They are not influenced by the environment, have mono or oligogenic genetic control, and practical, low-cost application, with no sophisticated equipment requirements (Rodrigues et al., 2010). However, they can be subjective, requiring an adequate training of evaluators. Aside from providing information on accessions, the use of descriptors allows for checking between-accession genetic diversity and is very helpful in accession's conservation and use. These analyses help to identify parents to be used in crop improvement programs (Cruz et al., 2011). They also allow for identifying groups with higher similarity on one side, emphasizing the need for new samples and the existence of duplicates on the other, which might be eliminated, reducing costs and workforce required for the conservation of accessions (Gonçalves et al., 2008). Genetic diversity is obtained through dissimilarity measures. Different methods might be employed and the choice of method depends on the type of descriptor, data collection method and the objectives of the researcher. For quantitative descriptors, Average Standardized Euclidean Distance (SED) and Mahalanobis Distance (MD) are the most frequently used methods (Mohhamadi and Prasanna, 2003). SED might be obtained through individual observations of accessions without the need for experiments that involve experimental designs. One application is the analysis of data derived from the multiplication of accessions. In these experiments, a large number of plants are cultivated in the field. To facilitate this 
study, accessions are cultivated in continuous rows without using experimental designs. During plant development and after fruit harvest, it is possible to collect a large amount of information and genetic diversity is obtained using SED.

However, SED considers neither the correlation between the traits studied nor experiment precision. When information derived from experimental assays is available, it is possible to obtain a residual dispersion matrix and the mean of traits. This information allows for estimating the Mahalanobis Distance (Cruz et al., 2012) since this method considers experimental error, this dissimilarity measure is influenced by the same factors that affect the error.

Hence, the more precise the trait estimation is, the lower is its residual variance, and consequently, the higher its contribution to the dissimilarity measure. However, in studies with germplasm, there might be high within-accession variations in traits, mainly with alogamous species, e.g. cucurbits. Such variations contribute to increase of residual variance, and consequently, to reduce the weight of certain variables in estimates of dissimilarity measures and might thus hinder accession differentiation.

There are also several methods to obtain dissimilarity measures for qualitative descriptors. One example is the Weighted Average Euclidean Distance (WED), which is used when accessions show high variability of multicategorical traits and when this variability has been quantified (Cruz et al., 2011).

Although both types of descriptors are indicated for the characterization of accessions, quantitative descriptors have been a priority due to their economic importance, easy measurement and data analysis. However, the exclusive use of this type of descriptor not always allows for a good separation of accessions. Syafii et al. (2015) studied the genetic diversity of maize accessions using quantitative descriptors based on Euclidean Distance. Syafii et al. (2009) and Rocha et al. (2009) characterized tomato accessions based on quantitative descriptors and calculated betweenaccession dissimilarity using the Mahalanobis Distance. By performing cluster analysis, these authors obtained clusters comprised by a large number of accessions. The use of qualitative descriptors combined with quantitative descriptors might improve the discrimination between accessions.

A survey of publications on vegetable genetic resources conducted by Sudré et al. (2007) revealed that qualitative data are poorly exploited in genetic diversity analyses and are frequently analyzed only by descriptive statistical procedures. In addition, when dissimilarity measures are calculated based on these descriptors, no joint analysis of qualitative and quantitative data is performed, especially in characterizations of more than one assay, which limits the interpretation of results.

Neitzkeet al. (2010) and Bento et al. (2007) characterized pepper accessions using qualitative and quantitative descriptors. However, the analysis of genetic diversity was performed separately for each set of descriptors, resulting in different clusters. Therefore, joint data analysis is required to obtain a better indication of the potential for preserved variability in germplasm banks (Torres et al., 2015; Sarkar et al., 2011).

Joint analysis of quantitative and qualitative descriptors might be performed using three methods. The first one is the conversion of descriptors into one single pattern. Quantitative traits might be converted into binary or multi-categorical qualitative descriptors using different methods. After being transformed, they might be analyzed together with qualitative descriptors and the dissimilarity matrix might be obtained using the adequate methodologies for this type of trait
(Martins et al., 2012). However, this method changes the nature of descriptors and their discriminating potential is thus decreased.

The second method is to adopt one single dissimilarity measure for all types of variables or different dissimilarity measures that have the same defining interval, e.g. Gower's Index (Cruz et al., 2011; Pavoine et al., 2009; Mohhamadi and Prasanna, 2003; Gower, 1971). However, for multicategorical qualitative variables, this index disregards the frequency of each class, i.e., it does not consider the existing within-accession variability.

The third alternative is to subdivide variables into groups (quantitative and binary, or multi-categorical qualitative descriptors) so that the most suitable dissimilarity measure is used for each group. With this procedure, several dissimilarity matrices are obtained, which might be analyzed separately or generate a joint dissimilarity matrix, whose elements are given by the mean of dissimilarities obtained through each dataset (Cruz et al., 2011). An improvement of this method is to use the sum of matrices, instead of the mean, as the latter might conceal the actual divergence between accessions.

Thus, the aim of this study was to apply different statistical analyses for quantitative and qualitative descriptors, using data on watermelon [Citrullus lanatus (Thunb.) Matsum \& Nakai] accessions characterized in different assays to identify the most suitable analysis to integrate descriptors in genetic diversity studies.

\section{Results and Discussion}

\section{Variability in watermelon accessions}

A wide morphological variability was observed between and within the accessions studied. Four descriptors did not meet ANOVA assumptions: two in experiment 1 (cotyledon width and pulp yield) and two in experiment 2 (seed emergence and length). Of the 24 quantitative descriptors submitted to ANOVA in each experiment, a significant between-accession difference was observed for 22 descriptors (Table 1). The ratio between the highest and the lowest mean square showed homogeneity of residual variances (Cruz et al., 2012); therefore, we performed a joint analysis of experiments. In this regard, there was a significant effect of the interaction genotype $\times$ environment on five of the quantitative variables, by which performing the joint analysis was possible (Table 1). However, as our objective was also to evaluate the clustering pattern of different strategies in each experiment, we also performed the separate analysis of variance. Mean variation coefficient (VC) was $17.8 \%$ in experiment 1 and $16.3 \%$ in experiment 2 (Table 1). High variation coefficients are typically found in morphological characterization experiments of watermelon accessions (Oliveira et al., 2008; Silva et al., 2006). This might be explained by the nature of quantitative traits, which are more sensitive to environmental variations (Rodrigues et al., 2010), but also by the existence of genetic variation within accessions. Fruit descriptors were the ones with the highest VC's (Table 1). Syafii et al. (2008) also observed high VC's for fruit traits, which might indicate that significant between-accession differences are more difficult to detect. The most uniform quantitative descriptors were seed descriptors (seed length, width, and thickness), indicating that these characteristics are the most uniform among accessions. Regarding qualitative descriptors, all of them showed polymorphism between and within accessions and also between the commercial varieties (witnesses) (Table 2). This variation might be influenced by different factors, 
Table 1. Means, significances of the effect of accessions, experimental variation coefficients (VC\%), and significance of interaction genotypes $\times$ environments $(\mathrm{G} \times \mathrm{E})$, for quantitative descriptors of 20 watermelon accessions and two commercial varieties (witnesses).

\begin{tabular}{|c|c|c|c|c|c|c|c|}
\hline Phase & Descriptor & Mean & $\begin{array}{c}\text { Experiment/Significance of } \\
\text { accessions }\end{array}$ & $\mathrm{VC} \%$ & $\begin{array}{l}\text { Mean } \\
\text { VC\% }\end{array}$ & $\begin{array}{l}\text { Significance of the } \\
\text { interaction } \mathrm{GxE}^{\mathrm{a}}\end{array}$ & $\begin{array}{c}\text { Mean } \\
\text { CV\%/phase }\end{array}$ \\
\hline \multirow{14}{*}{$\begin{array}{l}\text { Emergence } \\
\text { and plantlet }\end{array}$} & \multirow{2}{*}{$\mathrm{E}(\%)$} & 66.82 & $1 * *$ & 20.51 & \multirow{2}{*}{20.10} & \multirow[t]{2}{*}{ 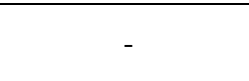 } & \multirow{14}{*}{14.2} \\
\hline & & 64.73 & 2 & 19.69 & & & \\
\hline & \multirow{2}{*}{ EVI } & 0.96 & $1 * *$ & 21.73 & \multirow{2}{*}{21.09} & \multirow{2}{*}{ ** } & \\
\hline & & 2.02 & $2 * *$ & 20.44 & & & \\
\hline & \multirow{2}{*}{ NDL } & 1.48 & $1 *$ & 23.30 & \multirow{2}{*}{20.95} & \multirow{2}{*}{ ns } & \\
\hline & & 1.38 & $2 * *$ & 18.59 & & & \\
\hline & \multirow{2}{*}{$\mathrm{HL}(\mathrm{cm})$} & 3.07 & $1 * *$ & 13.37 & \multirow{2}{*}{13.30} & \multirow[b]{2}{*}{ ns } & \\
\hline & & 3.70 & $2 * *$ & 13.22 & & & \\
\hline & \multirow{2}{*}{$\mathrm{HD}(\mathrm{mm})$} & 2.45 & $1 * *$ & 6.38 & \multirow{2}{*}{7.91} & \multirow{2}{*}{ ns } & \\
\hline & & 2.49 & $2 * *$ & 9.44 & & & \\
\hline & \multirow{2}{*}{$\mathrm{CL}(\mathrm{mm})$} & 32.02 & $1 * *$ & 7.56 & \multirow{2}{*}{7.81} & & \\
\hline & & 34.36 & $2 * *$ & 8.06 & & ns & \\
\hline & $\mathrm{CW}(\mathrm{mm})$ & 19.68 & 1 & 8.72 & & & \\
\hline & $\mathrm{cw}(\mathrm{mm})$ & 19.51 & $2 * *$ & 7.19 & 1.90 & - & \\
\hline & & 30.77 & $1^{\mathrm{ns}}$ & 7.43 & & & \\
\hline Flowering & NDUFMIF & 27.24 & $2 * *$ & 8.50 & 1.91 & ns & \\
\hline Flowering & & 35.77 & $1^{*}$ & 9.08 & & & 8.3 \\
\hline & NDOFFF & 34.33 & $2 * *$ & 8.27 & 8.68 & ns & \\
\hline & $\mathrm{FM}(\mathrm{k} \sigma)$ & 1.91 & $1 * *$ & 33.97 & 2802 & $* *$ & \\
\hline & ГIVI (Kg) & 3.68 & $2 * *$ & 23.87 & 20.92 & es & \\
\hline & $P M(k g)$ & 1.17 & $1 * *$ & 35.27 & 3246 & & \\
\hline & PMI (Kg) & 1.75 & $2 * *$ & 29.64 & 32.40 & ns & \\
\hline & $\mathrm{SM}(\mathrm{kg})$ & 0.71 & $1 * *$ & 36.28 & & & \\
\hline Fruit & $\mathrm{SIVI}(\mathrm{Kg})$ & 1.93 & $2 * *$ & 27.22 & 31.15 & ns & \\
\hline Fruit & $C D(\mathrm{~cm})$ & 11.85 & $1 * *$ & 11.15 & & & 22.0 \\
\hline & $\mathrm{CD}(\mathrm{cm})$ & 15.23 & $2 * *$ & 7.93 & 9.54 & ns & \\
\hline & & 24.21 & $1 * *$ & 15.38 & & & \\
\hline & $\mathrm{LD}(\mathrm{cm})$ & 31.28 & $2 * *$ & 13.29 & 14.34 & ns & \\
\hline & & 0.93 & $1^{*}$ & 22.07 & & & \\
\hline & $\operatorname{MS~I}(\mathrm{cm})$ & 1.64 & $2 * *$ & 15.65 & 18.80 & ns & \\
\hline & & 60.19 & 1 & 14.82 & & - & \\
\hline & PY (\%) & 47.00 & $2 * *$ & 17.23 & 16.03 & - & \\
\hline & TTA $(\%)$ & 1.31 & $1^{\mathrm{ns}}$ & 23.09 & 2301 & $n_{c}$ & \\
\hline Puln guality & $11 \mathrm{~A}(\%)$ & 1.07 & $2^{\mathrm{ns}}$ & 22.92 & 23.01 & ns & 170 \\
\hline Puip quanty & & 7.23 & $1 * *$ & 9.94 & & & 17.0 \\
\hline & SS ( Br1X) & 6.24 & $2 * *$ & 14.54 & 12.24 & w & \\
\hline & $\mathrm{VC}(\mathrm{mg} / 100$ & 8.71 & $1 *$ & 15.49 & 1685 & $* *$ & \\
\hline & $\mathrm{mL})$ & 10.46 & $2^{\mathrm{ns}}$ & 18.21 & 10.85 & wn & \\
\hline & $\mathrm{M} 100 \mathrm{~S}(\mathrm{~g})$ & 6.69 & $1 * *$ & 10.81 & 1165 & ns & \\
\hline & lNHOUs (g) & 8.29 & $2 * *$ & 12.49 & 11.05 & ns & \\
\hline & $\operatorname{TSM}(\mathrm{g})$ & 18.60 & $1^{*}$ & 41.52 & 32.00 & & \\
\hline & 1SIVI (g) & 37.41 & $2 * *$ & 22.48 & 32.00 & ns & \\
\hline & NSF & 269.80 & $1 *$ & 34.76 & 2726 & ns & \\
\hline & $\mathrm{NSF}$ & 439.00 & $2 *$ & 19.76 & 21.26 & ns & \\
\hline & & 28.76 & $1 * *$ & 35.82 & & $* *$ & \\
\hline Seeds & NSIUUP & 31.11 & $2 * *$ & 42.99 & 39.41 & w & 17.9 \\
\hline & & 2.11 & $1 * *$ & 6.24 & & & \\
\hline & $\mathrm{SI}(\mathrm{mm})$ & 2.24 & $2 * *$ & 5.96 & 0.10 & ns & \\
\hline & $\mathrm{SL}(\mathrm{mm})$ & 10.77 & $1 * *$ & 4.81 & 535 & - & \\
\hline & $\mathrm{SL}$ (IIIII) & 11.40 & 2 & 5.89 & $3.3 J$ & - & \\
\hline & & 6.55 & $1 * *$ & 4.62 & 401 & & \\
\hline & $\mathrm{SW}(\mathrm{mm})$ & 7.04 & $2 * *$ & 3.39 & 4.01 & ns & \\
\hline
\end{tabular}

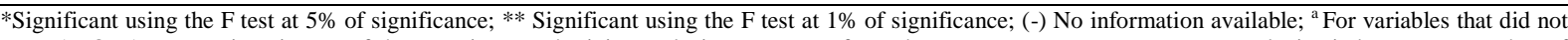
meet ANOVA assumptions in one of the experiments, the joint analysis was not performed; E: emergence; EVI: emergence velocity index; NDL: number of definitive leaves; HL: hypocotyl length; HD: hypocotyl diameter; CL: cotyledon length; CW: cotyledon width; NDOFMF: number of days for the opening of the first male flower; NDOFFF: number of days for the opening of the first female flower; FM: fruit mass; PM: pulp mass; SM: skin mass; CD: cross-section diameter of the fruit; LD: longitudinal diameter of the fruit; MST: mean skin thickness; PY: pulp yield; TTA: total titratable acidity; SS: soluble solids of the pulp; VCC: Vitamin C content; M100S: mass of 100 seeds; TSM: total seed mass; NSF: number of seeds per fruit; NS100P: number of seeds per $100 \mathrm{~g}$ of pulp; ST: seed thickness; SL: seed length; and SW: seed width. 
Table 2. Number of classes observed in 23 qualitative descriptors in 20 watermelon accessions and two commercial varieties (witnesses).

\begin{tabular}{|c|c|c|c|c|c|c|c|c|c|c|c|c|c|c|c|c|c|c|c|c|c|c|c|c|}
\hline Accession & PLD & SLD & LC & SAP & SPS & PBC & SSP & IC & SS & FL & PPC & SPC & CPS & $\mathrm{SC}$ & ISC & PP & UP & PC & PS & TS & CS & PC & $\mathrm{CC}$ & $\sum$ of classes \\
\hline 1 & 1 & 4 & 3 & 3 & 3 & 2 & 4 & 2 & 2 & 3 & 3 & 3 & 3 & 2 & 3 & 2 & 3 & 2 & 2 & 3 & 3 & 2 & 2 & 60 \\
\hline 2 & 1 & 4 & 2 & 3 & 3 & 2 & 4 & 2 & 2 & 4 & 3 & 4 & 3 & 2 & 3 & 2 & 3 & 3 & 2 & 3 & 3 & 2 & 4 & 64 \\
\hline 3 & 1 & 3 & 3 & 3 & 3 & 2 & 4 & 2 & 1 & 3 & 3 & 3 & 4 & 3 & 3 & 2 & 2 & 2 & 2 & 2 & 2 & 2 & 2 & 57 \\
\hline 4 & 1 & 4 & 2 & 3 & 3 & 3 & 2 & 2 & 1 & 3 & 3 & 4 & 3 & 2 & 3 & 2 & 3 & 3 & 2 & 2 & 2 & 1 & 1 & 55 \\
\hline 5 & 1 & 4 & 2 & 4 & 3 & 2 & 3 & 2 & 2 & 3 & 4 & 3 & 4 & 1 & 2 & 1 & 1 & 1 & 1 & 1 & 1 & 1 & 1 & 48 \\
\hline 7 & 1 & 3 & 2 & 4 & 3 & 3 & 3 & 2 & 2 & 3 & 3 & 4 & 3 & 2 & 3 & 2 & 2 & 3 & 2 & 2 & 2 & 2 & 3 & 59 \\
\hline 8 & 1 & 4 & 2 & 3 & 3 & 2 & 2 & 1 & 1 & 3 & 4 & 4 & 4 & 2 & 2 & 2 & 2 & 2 & 2 & 3 & 3 & 2 & 2 & 56 \\
\hline 11 & 1 & 3 & 2 & 3 & 3 & 1 & 3 & 2 & 2 & 3 & 2 & 4 & 3 & 2 & 2 & 2 & 3 & 2 & 2 & 2 & 3 & 2 & 2 & 54 \\
\hline 12 & 1 & 4 & 3 & 3 & 3 & 1 & 5 & 2 & 1 & 5 & 3 & 5 & 2 & 2 & 3 & 2 & 3 & 2 & 2 & 3 & 3 & 2 & 2 & 62 \\
\hline 15 & 1 & 4 & 3 & 3 & 3 & 3 & 6 & 2 & 2 & 2 & 3 & 3 & 4 & 2 & 3 & 2 & 3 & 3 & 2 & 4 & 4 & 2 & 3 & 67 \\
\hline 17 & 1 & 3 & 1 & 3 & 2 & 3 & 5 & 1 & 1 & 3 & 4 & 5 & 2 & 2 & 3 & 1 & 3 & 2 & 2 & 3 & 3 & 1 & 2 & 56 \\
\hline 18 & 1 & 3 & 2 & 3 & 3 & 1 & 4 & 2 & 2 & 3 & 3 & 3 & 4 & 2 & 3 & 2 & 3 & 2 & 2 & 3 & 3 & 2 & 1 & 57 \\
\hline 27 & 1 & 4 & 2 & 4 & 3 & 3 & 6 & 1 & 1 & 5 & 2 & 4 & 3 & 3 & 3 & 2 & 2 & 3 & 2 & 3 & 3 & 2 & 3 & 65 \\
\hline 33 & 2 & 4 & 2 & 3 & 3 & 3 & 6 & 2 & 2 & 5 & 3 & 4 & 2 & 3 & 3 & 1 & 1 & 1 & 2 & 2 & 3 & 1 & 1 & 59 \\
\hline 34 & 1 & 4 & 2 & 3 & 3 & 1 & 1 & 2 & 2 & 2 & 2 & 3 & 3 & 2 & 3 & 2 & 3 & 2 & 2 & 2 & 3 & 2 & 1 & 51 \\
\hline 36 & 3 & 4 & 2 & 4 & 3 & 1 & 5 & 2 & 2 & 4 & 4 & 4 & 5 & 3 & 3 & 2 & 3 & 2 & 2 & 3 & 4 & 2 & 4 & 71 \\
\hline 40 & 1 & 4 & 2 & 3 & 3 & 1 & 3 & 1 & 1 & 2 & 4 & 4 & 3 & 2 & 3 & 2 & 3 & 3 & 2 & 2 & 2 & 2 & 4 & 57 \\
\hline 41 & 1 & 3 & 2 & 3 & 3 & 1 & 4 & 2 & 1 & 3 & 3 & 4 & 3 & 3 & 3 & 2 & 3 & 2 & 2 & 2 & 2 & 2 & 2 & 56 \\
\hline 42 & 1 & 4 & 2 & 3 & 3 & 2 & 3 & 2 & 2 & 3 & 3 & 3 & 4 & 3 & 3 & 2 & 3 & 2 & 2 & 3 & 3 & 2 & 2 & 60 \\
\hline 46 & 1 & 4 & 3 & 4 & 3 & 2 & 4 & 1 & 1 & 3 & 4 & 4 & 4 & 3 & 3 & 2 & 3 & 3 & 1 & 1 & 1 & 2 & 3 & 60 \\
\hline Crimson Sweet & 1 & 4 & 2 & 3 & 2 & 1 & 1 & 1 & 1 & 3 & 3 & 2 & 1 & 2 & 2 & 1 & 1 & 1 & 2 & 2 & 2 & 2 & 2 & 42 \\
\hline Charleston Gray & 1 & 4 & 2 & 4 & 3 & 1 & 2 & 1 & 1 & 3 & 2 & 1 & 2 & 2 & 3 & 2 & 2 & 2 & 2 & 3 & 4 & 2 & 2 & 51 \\
\hline$\sum$ of classes & 25 & 82 & 48 & 72 & 64 & 41 & 80 & 37 & 33 & 71 & 68 & 78 & 69 & 50 & 62 & 40 & 55 & 48 & 42 & 54 & 59 & 40 & 49 & \\
\hline
\end{tabular}

PLD: primary lobulation degree; SLD: secondary lobulation degree; LC: leaf color; SAP: shape of the apical part; SPS: size of pistil scar; PBC: predominant background color; SSP: skin stripe pattern; IC: internal collapse; FL: free leaves; FS: fruit shape, PPC: predominant pulp color; SPC: secondary pulp color; CPS: color of the pulp around seeds; SC: seed color; ISC: intensity of seed color; PP: presence of pleurogram; UP: uniformity of pleurogram; PC: pleurogram color; PS: presence of stripes; TS: type of stripe; SC: stripe color; PC: presence of channels; and CC: color of channels. 


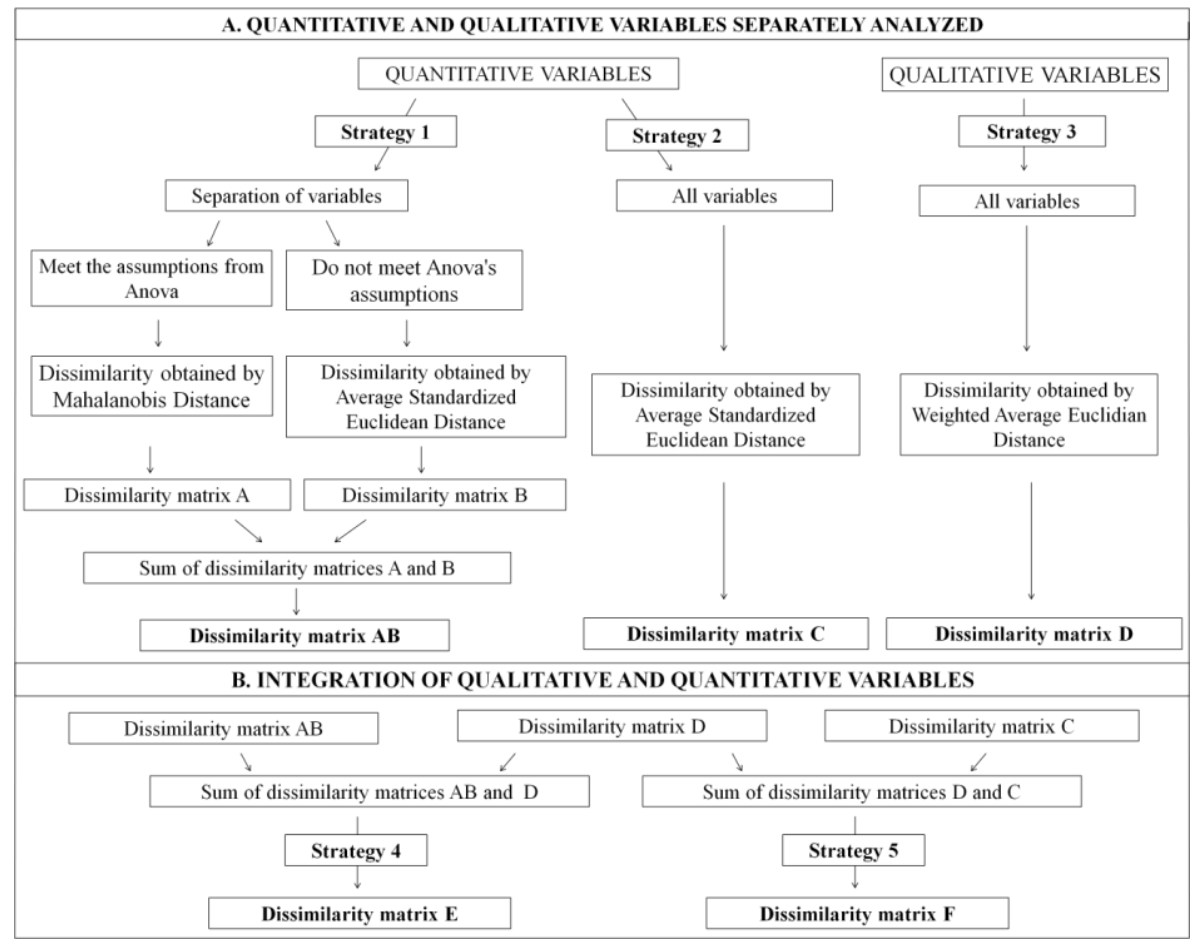

Fig 1. Flowchart of calculation of genetic dissimilarity matrices using 5 different data analysis strategies. A: Strategies for the separate analysis of quantitative and qualitative variables. B: Strategies for the integration of quantitative and qualitative variables.

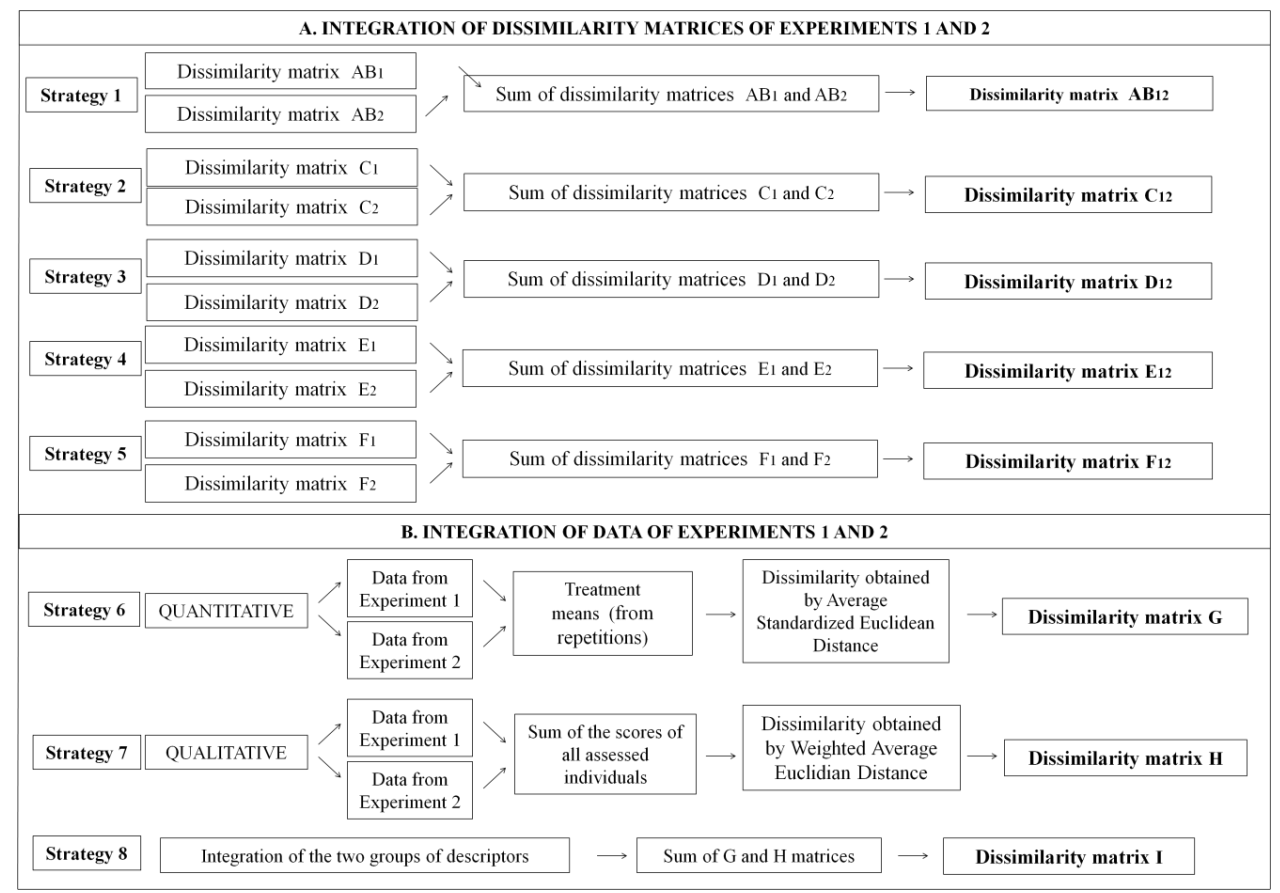

Fig 2. Flowchart of the analysis of joint diversity of two experiments for the characterization of watermelon accessions. A: Dissimilarity matrix integration of two experiments obtained using 5 different strategies. B: Integration of data from two experiments to obtain joint dissimilarity matrices. 
Table 3. Clusters with and without witnesses generated by five different strategies of genetic diversity analysis in watermelon accessions cultivated during rainy season (Experiment 1 ).

\begin{tabular}{|c|c|c|c|c|c|}
\hline Group. & $\mathrm{G}$ & Accessions which belong to the group & Group. & $\mathrm{G}$ & Accessions which belong to the group \\
\hline \multirow{4}{*}{$\mathrm{AB}_{\mathrm{t1}}{ }^{\mathrm{a}}$} & 1 & $\begin{array}{l}4,7,18,3,2,5,36,15,11,46,42,34,8,1,33,27, \\
41,17, \mathrm{CG}\end{array}$ & \multirow{4}{*}{$\mathrm{AB}_{1}$} & 1 & $4,7,18,3,2,5,36,15,11,46,42,34,8,1,33,27$ \\
\hline & 2 & $12, \mathrm{CS}$ & & 2 & 17,41 \\
\hline & 3 & 40 & & 3 & 40 \\
\hline & & & & 4 & 12 \\
\hline \multirow{6}{*}{$\mathrm{C}_{\mathrm{t} 1}$} & 1 & $\begin{array}{l}3,15,4,2,5,18,1,17,7,36,42,11,33,34,46,8, \\
41,40,27, \mathrm{CG}\end{array}$ & \multirow{6}{*}{$\mathrm{C}_{1}$} & 1 & $3,15,4,2,5,18,1,17,7,36,42,11,33,34$ \\
\hline & 2 & 12 & & 2 & 41,46 \\
\hline & 3 & CS & & 3 & 40 \\
\hline & & & & 4 & 8 \\
\hline & & & & 5 & 27 \\
\hline & & & & 6 & 12 \\
\hline \multirow{9}{*}{$D_{t 1}$} & 1 & $5,8,36,11,33, \mathrm{CG}$ & \multirow{9}{*}{$\mathrm{D}_{1}$} & 1 & 5,8 \\
\hline & 2 & $7,27,17,4,2,1$ & & 2 & $7,27,17,4,2,1$ \\
\hline & 3 & $15,40,12,42$ & & 3 & $33,46,36$ \\
\hline & 4 & 18,34 & & 4 & 15,41 \\
\hline & 5 & 41 & & 5 & 12,42 \\
\hline & 6 & 3 & & 6 & 18,34 \\
\hline & 7 & $\mathrm{CS}$ & & 7 & 41 \\
\hline & 8 & 46 & & 8 & 3 \\
\hline & & & & 9 & 11 \\
\hline \multirow{8}{*}{$\mathrm{E}_{\mathrm{t} 1}$} & 1 & $\begin{array}{l}2,4,7,18,3,27,15,42,1,17,41,40,36,34,46,33 \text {, } \\
11,5, \mathrm{CG}\end{array}$ & \multirow{8}{*}{$\mathrm{E}_{1}$} & 1 & $2,4,7,18,3,27,15,42$ \\
\hline & 2 & 12 & & 2 & $5,36,33,11,46$ \\
\hline & 3 & 8 & & 3 & 17,41 \\
\hline & 4 & $\mathrm{CS}$ & & 4 & 1 \\
\hline & & & & 5 & 40 \\
\hline & & & & 6 & 34 \\
\hline & & & & 7 & 12 \\
\hline & & & & 8 & 8 \\
\hline \multirow{6}{*}{$\mathrm{F}_{\mathrm{t} 1}$} & 1 & $\begin{array}{l}5,8,36,11,33,46,7,42,18,40,15,12,4,2,17,41, \\
27,3,34,1, \mathrm{CS}\end{array}$ & \multirow{6}{*}{$\mathrm{F}_{1}$} & 1 & $5,8,36,11,33$ \\
\hline & 2 & $\mathrm{CG}$ & & 2 & $4,17,2,7,18,40,41,42,15,3$ \\
\hline & & & & 3 & 12,27 \\
\hline & & & & 4 & 1 \\
\hline & & & & 5 & 34 \\
\hline & & & & 6 & 46 \\
\hline
\end{tabular}

${ }^{\mathrm{a}}$ t: Clusters with witnesses; $\mathrm{AB}_{1}$ : Clusters of quantitative descriptors using strategy 1 (MD and $\mathrm{SED}$ ); $\mathrm{C}_{1}$ : Clusters of quantitative descriptors using strategy 2 (SED); $\mathrm{D}_{1}$ : Clusters of qualitative descriptors using strategy $3(\mathrm{WED}) ; \mathrm{E}_{1}$ : Cluster obtained by integrating quantitative and qualitative descriptors using strategy $4\left(\mathrm{AB}_{1}+\mathrm{D}_{1}\right) ; \mathrm{F}_{1}$ : Cluster obtained by integrating quantitative and qualitative descriptors using strategy $5\left(C_{1}+D_{1}\right)$.

Table 4. Clusters with and without witnesses generated by five different strategies of genetic diversity analysis in watermelon accessions cultivated during dry season (Experiment 2).

\begin{tabular}{|c|c|c|c|c|c|}
\hline Group. & $\mathrm{G}$ & Accessions which belong to the group & Group. & $\mathrm{G}$ & Accessions which belong to the group \\
\hline \multirow{6}{*}{$\mathrm{AB}_{\mathrm{t} 2}{ }^{\mathrm{a}}$} & 1 & $\begin{array}{c}11,41,18,2,7,36,3,42,33,5,34,46,17, \\
\text { CG }\end{array}$ & \multirow{6}{*}{$\mathrm{AB}_{2}$} & 1 & $\begin{array}{c}11,41,18,2,7,36,3,42,33,5,34,46,17 \\
1\end{array}$ \\
\hline & 2 & $12, \mathrm{CS}$ & & 2 & 8,15 \\
\hline & 3 & $1,4,8,15$ & & 3 & 4 \\
\hline & 4 & 40 & & 4 & 40 \\
\hline & \multirow[t]{2}{*}{5} & 27 & & 5 & 12 \\
\hline & & & & 6 & 27 \\
\hline \multirow{6}{*}{$\mathrm{C}_{\mathrm{t} 2}$} & 1 & $\begin{array}{c}8,34,1,2,4,3,18,46,41,15,7,36,5,11, \\
33\end{array}$ & \multirow{6}{*}{$\mathrm{C}_{2}$} & 1 & $\begin{array}{c}8,34,1,2,4,3,18,46,41,15,7,36,5,11, \\
33,42,17\end{array}$ \\
\hline & 2 & 40,42 & & 2 & 27 \\
\hline & 3 & $12, \mathrm{CS}$ & & 3 & 40 \\
\hline & 4 & CG & & 4 & 12 \\
\hline & 5 & 27 & & & \\
\hline & 6 & 17 & & & \\
\hline \multirow{6}{*}{$\mathrm{D}_{\mathrm{t} 2}$} & 1 & $7,17,2,46,40,4,1,15,11,8,18,41$ & \multirow{6}{*}{$\mathrm{D}_{2}$} & 1 & $7,17,2,46,40,4,1,15,11,8,18,41$ \\
\hline & 2 & $12,27,3,42,33$ & & 2 & $12,27,3,42,33$ \\
\hline & 3 & CS , CG & & 3 & 36 \\
\hline & 4 & 5 & & 4 & 5 \\
\hline & 5 & 36 & & 5 & 34 \\
\hline & 6 & 34 & & & \\
\hline \multirow{5}{*}{$\mathrm{E}_{\mathrm{t} 2}$} & 1 & $1,4,2,41,18,11$ & \multirow{5}{*}{$E_{2}$} & 1 & $1,4,2,41,18,11,36,46,7,17,40$ \\
\hline & 2 & $3,42,33$ & & 2 & $3,42,33,27,12$ \\
\hline & 3 & 7,17 & & 3 & $8,34,15$ \\
\hline & 4 & $8,34,15$ & & 4 & 5 \\
\hline & 5 & $12, \mathrm{CS}$ & & 5 & 12 \\
\hline
\end{tabular}




\begin{tabular}{cccccc} 
& 6 & 36,46 & & & \\
& 7 & $5, \mathrm{CG}$ & & & \\
& 8 & 40 & & & \\
& 9 & 27 & 2 & $1,4,2,46,18,15,8,34,41,11,7,36$ \\
& 1 & $1,4,2,46,18,15,8,34,41,1,7,36,17$ & 3 & $3,42,33,27,12$ \\
$\mathrm{~F}_{\mathrm{t} 2}$ & $3,42,33,27,12$ & $\mathrm{~F}_{2}$ & 3 & 5 \\
& 2 & $\mathrm{CS}, \mathrm{CG}$ & 4 & 17 \\
& 3 & 5 & & 5 & 17 \\
\hline
\end{tabular}

${ }^{\mathrm{a}}$ t: Clusters with witnesses; $\mathrm{AB}_{2}$ : Clusters of quantitative descriptors using strategy 1 (MD and SED); $\mathrm{C}_{2}$ : Clusters of quantitative descriptors using strategy 2 (SED); $\mathrm{D}_{2}$ : Clusters of qualitative descriptors using strategy 3 (WED); $E_{2}$ : Clusters obtained by integrating quantitative and qualitative descriptors using strategy $4\left(A B_{2}+D_{2}\right) ; F_{2}$ : Clusters obtained by integrating quantitative and qualitative descriptors using strategy $5\left(C_{2}+D_{2}\right)$.

Table 5. Dissimilarity between clusters generated by five strategies for data analysis in two characterization experiments of watermelon accessions.

\begin{tabular}{lll}
\hline Experiment & Clusters with witnesses & Clusters without witnesses \\
\hline \multirow{2}{*}{1} & Group 1: $\mathrm{AB}_{\mathrm{t} 1}{ }^{\mathrm{a}}, \mathrm{C}_{\mathrm{t} 1}, \mathrm{E}_{\mathrm{t} 1}, \mathrm{~F}_{\mathrm{t} 1}$ & Group 1: $\mathrm{AB}_{1}, \mathrm{C}_{1}, \mathrm{E}_{1}$ \\
& Group 2: $\mathrm{D}_{\mathrm{t} 1}$ & Group 2: $\mathrm{D}_{1}, \mathrm{~F}_{1}$ \\
\hline \multirow{2}{*}{2} & Group 1: $\mathrm{AB}_{\mathrm{t} 2}{ }^{\mathrm{b}}, \mathrm{C}_{\mathrm{t} 2}, \mathrm{D}_{\mathrm{t} 2}, \mathrm{~F}_{\mathrm{t} 2}$ & Group 1: $\mathrm{AB}_{2}, \mathrm{C}_{2}$ \\
& Group 2: $\mathrm{E}_{\mathrm{t} 2}$ & Group 2: $\mathrm{D}_{2}, \mathrm{E}_{2}, \mathrm{~F}_{2}$ \\
\hline \multirow{2}{*}{1 and 2 } & Group 1: $\mathrm{AB}_{\mathrm{t} 1}, \mathrm{AB}_{\mathrm{t} 2}, \mathrm{C}_{\mathrm{t} 1}, \mathrm{C}_{\mathrm{t} 2}, \mathrm{D}_{\mathrm{t} 2}, \mathrm{E}_{\mathrm{t} 1}, \mathrm{~F}_{\mathrm{t} 1}, \mathrm{~F}_{\mathrm{t} 2}$ & Group 1: $\mathrm{AB}_{1}, \mathrm{AB}_{2}, \mathrm{C}_{1}, \mathrm{C}_{2}, \mathrm{D}_{2}, \mathrm{E}_{1}, \mathrm{E}_{2}, \mathrm{~F}_{2}$ \\
& Group 2: $\mathrm{E}_{\mathrm{t} 2}$ & Group 2: $\mathrm{D}_{1}, \mathrm{~F}_{1}$ \\
& Group 3: $\mathrm{D}_{\mathrm{t} 1}$ & \\
\hline
\end{tabular}

${ }^{\mathrm{a}}$ Experiment $1 ;{ }^{\mathrm{b}}$ Experiment 2; t: Clusters with witnesses; AB: Clusters of quantitative descriptors using strategy 1 (MD and SED); C: Clusters of quantitative descriptors using strategy 2 (SED); D1: Clusters of qualitative descriptors using strategy 3 (WED); E1: Cluster obtained by integrating quantitative and qualitative descriptors using strategy $4\left(\mathrm{AB}_{1}+\mathrm{D}\right)$; F: Cluster obtained by integrating quantitative and qualitative descriptors using strategy $5(\mathrm{C}+\mathrm{D})$.

Table 6. Clusters of 20 watermelon accessions resulting from the joint analysis of two experiments, based on eight different strategies.

\begin{tabular}{|c|c|c|c|c|c|}
\hline Group. & G & Accessions which belong to the group & Group. & $\mathrm{G}$ & Accessions which belong to the group \\
\hline \multirow{6}{*}{$\mathrm{AB}_{12}{ }^{\mathrm{a}}$} & 1 & $\begin{array}{c}7,36,2,18,3,11,5,42,46,4,34,15,1,41 \\
33,8,17\end{array}$ & \multirow{6}{*}{$\mathrm{F}_{12}$} & 1 & $7,17,2,4,41,18,15,1$ \\
\hline & 2 & 40 & & 2 & $11,36,46,8$ \\
\hline & 3 & 27 & & 3 & $12,42,3,27$ \\
\hline & 4 & 12 & & 4 & 5,33 \\
\hline & & & & 5 & 34 \\
\hline & & & & 6 & 40 \\
\hline \multirow{4}{*}{$\mathrm{C}_{12}$} & 1 & $\begin{array}{c}8,34,2,18,3,4,15,41,46,5,7,36,11,33 \\
17,42,1\end{array}$ & \multirow{4}{*}{$\mathrm{G}_{12}$} & 1 & $7,36,17,15,41,1,4,2,3,18,5,46,11,34,42,33,8$ \\
\hline & 2 & 40 & & 2 & 40 \\
\hline & 3 & 12 & & 3 & 27 \\
\hline & 4 & 27 & & 4 & 12 \\
\hline \multirow{6}{*}{$\mathrm{D}_{12}$} & 1 & $7,17,2,4,1,40,41,15,27,18,42$ & \multirow{6}{*}{$\mathrm{H}_{12}$} & 1 & $\begin{array}{c}1,2,4,15,40,17,7,41,18,3,46,27,42,11,12,34,8, \\
36\end{array}$ \\
\hline & 2 & 3,12 & & 2 & 5,33 \\
\hline & 3 & $8,11,36,46$ & & & \\
\hline & 4 & 33 & & & \\
\hline & 5 & 5 & & & \\
\hline & 6 & 34 & & & \\
\hline \multirow{7}{*}{$\mathrm{E}_{12}$} & 1 & $1,4,2,7,18,17,41,15,3,42$ & \multirow{7}{*}{$\mathrm{I}_{12}$} & 1 & $1,4,2,18,15,41,17,7,3,46,11,36,42,34$ \\
\hline & 2 & $36,46,11,33$ & & 2 & 27,33 \\
\hline & 3 & 8,34 & & 3 & 12 \\
\hline & 4 & 40 & & 4 & 5 \\
\hline & 5 & 5 & & 5 & 8 \\
\hline & 6 & 27 & & 6 & 40 \\
\hline & 7 & 12 & & & \\
\hline
\end{tabular}

${ }^{a} \mathrm{AB}_{12}$ : Clusters of quantitative descriptors using strategy 1 (MD and SED); $\mathrm{C}_{12}$ : Clusters of quantitative descriptors using strategy 2 (SED); $\mathrm{D}_{12}:$ Clusters of qualitative descriptors using strategy 3 (WED); $\mathrm{E}_{12}$ : Cluster obtained by integrating quantitative and qualitative descriptors using strategy $4\left(\mathrm{AB}_{12}+\mathrm{D}_{12}\right) ; \mathrm{F}_{12}$ : Cluster obtained by integrating quantitative and qualitative descriptors using strategy $5\left(\mathrm{C}_{12}+\mathrm{D}_{12}\right)$; G: Clusters of quantitative descriptors obtained by strategy 6 (overall mean of accessions considering all individuals in both experiments, dissimilarity obtained by SED); H: Cluster of qualitative descriptors using strategy 7 (dissimilarity matrix based on the scores of all individuals evaluated, obtained by WED); I: Integration of quantitative and qualitative descriptors using strategy $8(\mathrm{G}+\mathrm{H})$.

Table 7. Dissimilarity between clusters generated by the joint analysis of data from two characterization experiments in watermelon accessions.

\begin{tabular}{lc}
\hline Group & Clusters \\
\hline 1 & $\mathrm{AB}_{12}{ }^{\mathrm{a}}, \mathrm{C}_{12}, \mathrm{G}, \mathrm{H}, \mathrm{I}$ \\
2 & $\mathrm{D}_{12}, \mathrm{E}_{12}$ \\
3 & $\mathrm{~F}_{12}$ \\
\hline $\mathrm{B}_{12}$ : Clusters of quantitative descriptors using strategy 1 (MD and SED); $\mathrm{C}_{12}:$ Clusters of quantitative descriptors using strategy $2(\mathrm{SED}) ; \mathrm{D}_{12}:$ Clusters of qualitative \\
scriptors using strategy 3 (WED); $\mathrm{E}_{12}:$ Cluster obtained by integrating quantitative and qualitative descriptors using strategy $4(\mathrm{AB}$ \\
(2)
\end{tabular}


such as: subjectivity of some qualitative descriptors, which might hinder the evaluation process; small environmental effects suffered by these descriptors; and culture management by farmers, who do not control pollinations and frequently mix and exchange seeds from different genotypes, which also contributes to the variability observed.

Out of 23 qualitative descriptors evaluated, there could be 23 to 82 classes within each accession. Among accessions, classes ranged from 48 to 71 . For the witnesses, Crimson Sweet and Charleston Gray, the number of classes were 42 and 51, respectively, thus emphasizing a higher variability in CG.

\section{Analysis of genetic diversity of accessions in each experiment}

The different strategies of genetic dissimilarity analysis in each experiment resulted in 20 clusters (Tables 3 and 4). Comparing the clusters generated within each experiment, we observed that accessions were clustered differently according to the strategy adopted. Syafii et al. (2011) reported that high differences between clusters might occur due to the biological response of accessions to different environments, and are not necessarily caused by the use of inadequate statistical methodologies. However, this differentiated cluster pattern between experiments reveals the need for investigating methods that minimize the effect of the interaction $\mathrm{G} \times \mathrm{E}$ on cluster patterns.

We observed differences in the methods for separation of accessions; however, due to the high number of clustering strategies, interpreting this difference proved complicated. Therefore, it was necessary to separately analyze dissimilarities between clusters generated in the analyses of each experiment to better analyze the consistency of each cluster strategy and similarity between strategies (Table 5).

Analyses of quantitative descriptors $\mathrm{AB}$ (MD and SED) and $C$ (SED) showed similar clusters in both experiments, with and without witnesses (Table 5). When comparing them in each experiment, clustering strategies $\mathrm{AB}$ and $\mathrm{C}$ with witnesses equally allocated 19 accessions in experiment 1 (Table 3 ) and equally allocated 15 accessions in experiment 2 (Table 4). When witnesses were removed, $\mathrm{AB}$ and $\mathrm{C}$ equally allocated 16 and 17 accessions in experiments 1 and 2, respectively.

When we evaluated commercial cultivars (witnesses) and accessions, witnesses typically formed separate clusters and accessions form one or few clusters. This segregation has already been observed between cultivar Crimson Sweet (CS) and accessions collected in Mossoró-RN and in three regions of Bahia, with data evaluated using graphic dispersion, the generalized Mahalanobis distance, and the UPGMA method (Oliveira et al., 2008; Silva et al., 2006).

Segregation between accessions and commercial cultivars is due to two factors. The first one is that most quantitative traits of commercial types are superior compared to accessions. The second one is the use of generalized Mahalanobis distance for dissimilarity calculation. This method interprets the existing within-accession variation as lack of precision, which minimizes the importance of these traits in dissimilarity calculation. As the commercial types (witnesses) are very uniform, they show higher dissimilarity values and thus tend to form isolated groups. In our study, this tendency was more evident in experiment 1 , which had a higher experimental variation coefficient (Table 1) in clusters $\mathrm{ABt}_{1}$ and $\mathrm{Ct}_{1}$ (Table 3). The use of different clustering strategies using quantitative descriptors made it possible for accessions to be clustered similarly in both experiments
(Table 5). The clustering pattern was maintained when strategies $\mathrm{AB}$ and $\mathrm{C}$ were compared between experiments 1 and 2 (Tables 3 and 4). Strategies $A B_{t 1}$ and $A_{t 2}, C_{t 1}$ and $C_{t 2}$ equally allocated 17 and 16 accessions in the presence of witnesses, and 14 and 17 accessions when witnesses were removed $\left(\mathrm{AB}_{1}\right.$ and $\mathrm{AB}_{2}, \mathrm{C}_{1}$ and $\left.\mathrm{C}_{2}\right)$, respectively. Such fact might have occurred due to both the absence of high variations in environmental conditions during the experiments and to good experimental precision. The cluster obtained by qualitative descriptors (D) differed from clusters obtained by quantitative descriptors ( $\mathrm{AB}$ and $\mathrm{C}$ ) in all situations, except for experiment 2 with witnesses $\left(D_{t 2}\right)$ (Table 5). In experiment 1 with or without witnesses, strategy $\mathrm{D}$ allocated only eight to nine accessions similarly to $\mathrm{AB}$ and $C$ (Table 3). On the other hand, the number of coincidences between $\mathrm{D}, \mathrm{AB}$, and $\mathrm{C}$ ranged from 9 to 12 (Table 4) in experiment 2 . In characterizations of pepper accessions, a similar situation was observed (Neitzke et al., 2010; Bento et al., 2007). This emphasizes the need for using the two groups of descriptors in germplasm characterization, since they access different regions of the plant genome. In addition, it reinforces the importance of integrating these groups for a more consistent analysis of the genetic diversity of accessions (Sudré et al., 2007).

Comparing the clusters generated from qualitative descriptors in both experiments (Table 5), matrices $D_{1}$ and $D_{2}$ were divergent as they clustered accessions differently. Only eight accessions were equally allocated by clusters $D_{t 1}$ and $D_{t 2}$ (with witnesses). When witnesses were removed, $D_{1}$ and $\mathrm{D}_{2}$ equally clustered nine accessions. Several factors might have contributed to this divergence in the clustering pattern generated using qualitative descriptors, such as high withinaccession variability (Table 2), small environmental effects that might influence qualitative descriptors, and the participation of different evaluators in each experiment (Ramalho et al., 2005). Within-accession variability might be detected by increasing the number of individuals in the plot, but this would result in increased workforce and area for conducting experiments, which is not always possible. Another aspect needs to be highlighted is the variation in environmental conditions, which is sometimes out of control. The caution must be taken to well-characterize the environment of each evaluation, allocate blocks to homogeneous soil strips, and to keep crops and phytosanitary treatments identical between assays. Aside from good training, evaluators are required to use well-elaborated photographic scales to decrease subjectivity in evaluations and to make it possible to isolate the factors that might influence the consistency of clusters when only using qualitative descriptors.

Clusters resulting from the sum of qualitative and quantitative descriptors (E and F) did not follow a clustering pattern, and they were sometimes allocated with clusters derived from quantitative descriptors ( $\mathrm{AB}$ and $\mathrm{C}$ ), and sometimes with qualitative descriptors (D) (Table 5).

Considering that dissimilarity matrices $\mathrm{E}$ and $\mathrm{F}$ are formed by two components, and because one of them is equal for both (component D-qualitative), the strategy which determines the difference between them is the one employed for the calculation of the dissimilarity between quantitative descriptors. As only two of the 26 descriptors in each experiment did not meet ANOVA assumptions (Table 1), the dissimilarity calculated by strategy $\mathrm{AB}$ is comprised of almost exclusively by the fraction calculated using the generalized Mahalanobis distance.

Thus, in experiment 1, the higher $\mathrm{VC}$ for quantitative descriptors reduced the dissimilarity between accessions in 
$E_{1}$, allocating them to the same group as $A_{1}$ and $C_{1}$. Since Euclidean distance suffers a much lower influence of withinaccession variation, cluster $F_{1}$ was allocated together with $D_{1}$. The lower variation in experiment 2 allocated clusters $E_{2}$, $F_{2}$, and $D_{2}$ to the same group (Table 5). This means that in experiments with a lower experimental variation coefficient, the use of average Standardized Euclidean distance tends to equally cluster accessions as opposed to the Mahalanobis Distance.

\section{Joint analysis of experiments}

With the purpose of obtaining a cluster that contemplates both assays, the joint analysis of experiments resulted in eight dissimilarity matrices and 16 clusters (presence and absence of witnesses). Again, witnesses influenced clustering patterns and, knowing that this tendency has already been observed in other studies (Oliveira et al., 2008; Silva et al., 2007; Souza et al., 2004), we performed analysis interpretation without witnesses to better visualize the between-accession variability in this study (Table 6).

Similarly to separate experiments, there was variation in group and accession allocation inside clusters. Interpreting this wide variation was easier when we performed the study of dissimilarity between these clusters using the method by Cole-Rodgers et al. (1997), forming three groups (Table 7).

Some patterns already observed in separate analyses, where maintained in the joint analysis of experiments. Thus, when we analyzed the clusters generated by the quantitative matrices $\mathrm{AB}_{12}$ and $\mathrm{C}_{12}$ without witnesses, we observed that they remained allocated to the same group. The cluster resulting from matrix $G$ (obtained by using the average Standardized Euclidean Distance applied to the overall means of quantitative variables from both experiments) was also allocated to the same group of matrices $\mathrm{AB}_{12}$ and $\mathrm{C}_{12}$. Cluster $\mathrm{H}$ was allocated to the same group, despite the fact that it resulted from a matrix that integrates only qualitative data.

Cluster $\mathrm{D}_{12}$ also maintained the same pattern observed in separate experiments, and it was allocated to a different group. It is worth noting that by using qualitative descriptors, there was a variation in clusters between experiment 1 and experiment 2 (Table 5), whether due to within-accession variations to the characterization environment, and/or to the low interaction between these factors. In this setting, $D_{12}$, which derived from the sum of matrices generated in separate experiments, contemplates these effects and corrects this influence as it discriminates accessions inside each experimental condition and sums up this dissimilarity, unlike matrix $\mathrm{H}$.

Matrices that integrate quantitative and qualitative data of both experiments are represented by the letters E, F, and I. These matrices generated different clustering patterns of accessions, since each one was allocated to separate groups. Matrix I results from the sum of matrices G (quantitative) and $\mathrm{H}$ (qualitative) and was allocated together with the group of predominantly quantitative descriptors. However, matrices $G$ and $\mathrm{H}$ did not contemplate the environmental effect. In other words, the relative performance of accessions according to environmental variations was concealed, as matrix $G$ results from the overall mean of quantitative variables in assays and matrix $\mathrm{H}$ considers all individuals evaluated as if they were in one single experiment. It is important to emphasize that $19 \%$ of quantitative descriptors showed significant genotype $\mathrm{x}$ environment interaction (Table 1), which explains the inconsistencies present in the clustering patterns that used these strategies.
To mitigate the effect of the interaction $\mathrm{G} \times \mathrm{E}$ on quantitative data and potential influences that already reported for qualitative data, matrices $\mathrm{E}$ and $\mathrm{F}$ stood out as important alternatives. However, some observations are in place, as clusters generated by these matrices $\left(E_{12}\right.$ and $\left.F_{12}\right)$ were also allocated to different groups.

In cluster $\mathrm{E}_{12}\left(\mathrm{AB}_{12}+\mathrm{D}_{12}\right)$, the dissimilarity of quantitative data was obtained using MD. As previously explained in the separate analysis, this methodology considers withinaccession variability with no precision, reducing the importance of these variables in total dissimilarity, causing $E_{12}$ to be allocated to the same group of qualitative descriptors represented by $\mathrm{D}_{12}$. Therefore, this strategy was not the most suitable one for this set of accessions, with high within-accession variability (Table 1). However, for a set of uniform accessions, e.g. autogamous plants or endogamous strains of alogamous or mixed plants, this is the most suitable strategy as it weighs each variable according to the precision, by which it was evaluated.

In cluster $F_{12}\left(C_{12}+D_{12}\right)$, quantitative data dissimilarity was obtained using SED. Although this dissimilarity measure does not consider the precision of data collected (Cruz et al., 2011), matrix $F_{12}$ contemplates the interaction $G \times E$, since it results from the sum of the matrices obtained for each experiment. Moreover, in each experiment, the means of quantitative data derive from experiments with local control, which also reduces the environmental effect in each experiment.

Thus, the cluster generated by matrix $F_{12}$ was the most suitable one for the group of accessions studied. Although this dissimilarity measure (SED) is indicated for experiments without experimental designs (Cruz et al., 2012; Mohhamadi and Prasanna, 2003), a design with local control is recommended for watermelon germplasm characterization with high within-accession variability. This is mainly in order to obtain means that are less influenced by the environment, and the use of SED is recommended to obtain a dissimilarity matrix between accessions.

The strategy $\mathrm{F}$ is typically used in accession diversity in principal component analysis (PCA), which shows advantages as it allows obtaining dissimilarity matrices based on both quantitative and qualitative descriptors using the most suitable methods (SED and WED) for each type of descriptor. In addition, Tocher's clustering allows for the partitioning of genotypes when the number of groups to be formed is not previously known, which also decreases subjectivity in the graphical analysis (PCA) of diversity.

For a better insight into what defines each group, a detailed study of the cluster generated by matrix $F_{12}$ will allow for the morphoagronomic characteristics. These accessions collected from the State of Rio Grande do Sul which to be well documented and subsidize their use in crop improvement programs. In addition, it shall allow for identifying groups with higher similarity; thus, emphasizing the need for new samples and the existence of replicates, which might be eliminated to reduce costs and the workforce required for the conservation of accessions (Cruz et al., 2011; Marim et al., 2009).

Strategy F might be used for the study of genetic diversity in other cucurbit species, such as melon and pumpkin, which might also show high within-accession genetic variability (Santos, 2015; Lima Neto, 2013; Priori et al., 2012; Buso et al., 2004; Silva et al., 1997). 


\section{Materials and Methods}

\section{Cultivation site and season}

The experiments were conducted at the Experimental Field of the Department of Technology and Social Sciences of the State University of Bahia (DTCS/UNEB) in the municipality of Juazeiro - BA $\left(09^{\circ} 24^{\prime} 50^{\prime \prime} \mathrm{S}\right.$; 40 $30^{\prime} 10^{\prime \prime} \mathrm{W} ; 368 \mathrm{~m}$ of altitude) in randomized blocks with three replicates, during two seasons: rainy, from December 2014 to March 2015 (97.0 $\mathrm{mm}$ of total rainfall) and dry, from April to July 2015 (38.0 $\mathrm{mm}$ of total rainfall). A total of 20 watermelon accessions collected in the State of Rio Grande do Norte, with two commercial cultivars as witnesses, Crimson Sweet (Feltrin Sementes Ltda.) and Charleston Gray (Sementes do Vale Ltda), were evaluated (Supplementary Table 1). Technical recommendations for this culture in the region have been adopted.

\section{Descriptors evaluated}

A total of 49 descriptors (26 quantitative and 23 qualitative) were applied and subdivided according to the phenological phases of the culture (Supplementary Table 2). In the seedlings phase, there were seven descriptors: emergence percentage, emergence velocity index, number of definitive leaves, hypocotyl length and diameter, cotyledon length and diameter. Two descriptors in the vegetative phase: degree of primary and secondary lobulation of the leaf and leaf color. Two in the reproductive phase: number of days for the opening of the first male flower and of the female flower. Twenty descriptors related to fruit: fruit mass, skin mass, pulp mass, mean skin thickness, cross-section and longitudinal diameter of the fruit, pulp yield, titratable acidity, soluble solids, vitamin $\mathrm{C}$ content, shape of the apical part, size of the pistil scar, predominant background color, skin strip pattern, internal collapse, presence of free seeds, fruit shape, predominant pulp color, secondary pulp color, and color of the pulp around the seeds. Seventeen descriptors related to seed: mass of 100 seeds, total seed mass, total number of seeds, seed length, width, and thickness, number of seeds in $100 \mathrm{~g}$ of pulp, color and intensity of seed color, presence, evenness and color of pleurogram, presence, type, and color of strips, presence and color of channels.

\section{Statistical analyses}

Statistical analyses were performed using the Genes Software for Genetics and Statistics (Cruz, 2013). Initially, we observed normality and homogeneity of quantitative variables; those that did not meet the assumptions of analysis of variance (ANOVA) were transformed. Variables that did not meet assumptions even after transformation were discarded from the analysis of variance. Separate and Joint analyses of variance were performed for both experiments.

Genetic diversity of accessions was obtained using eight different strategies, which varied according to the nature of the variable, whether or not they met ANOVA assumptions, and separate or joint analysis of the experiments. The term "strategy" refers to a group of statistical procedures used to analyze data.

\section{Separate analysis of experiments}

Dissimilarity matrices between accessions were obtained using five different strategies for each experiment. For quantitative variables, two strategies were used based on whether they met ANOVA assumptions or not.

In strategy 1 , the analysis of variance was performed for quantitative variables with normal distribution. For these variables, dissimilarity matrix A was obtained by using the Mahalanobis Distance (MD). For variables that did not meet ANOVA assumptions, dissimilarity matrix B was obtained using the Average Standardized Euclidean Distance (SED). Afterwards, matrices A and B were summed up, resulting in matrix $\mathrm{AB}$. In strategy 2 , there was no separation of quantitative variables and dissimilarity matrix $\mathrm{C}$ was obtained using SED (Figure 1).

For qualitative variables, we used the method of Weighted Average Euclidean Distance (WED) to obtain dissimilarity matrix D, and this process was called Strategy 3.

The interaction between different types of descriptors (quantitative and qualitative) was performed as follows (Figure 1); using the sum of matrices $\mathrm{AB}$ and $\mathrm{D}$, resulting in dissimilarity matrix E (Strategy 4), and by the sum of matrices $\mathrm{C}$ and $\mathrm{D}$, resulting in dissimilarity matrix $\mathrm{F}$ (Strategy 5). Although joint dissimilarity matrix is reportedly obtained by taking the average of individual dissimilarities (Cruz et al., 2011), we applied the sum of individual matrices. This is because one inconvenience of using the mean of matrices is that the discriminating potential of accessions in each individual matrix might be concealed by their mean and there is a risk of obtaining a cluster pattern that is not compatible with the genetic diversity of accessions.

After obtaining each dissimilarity matrix $(\mathrm{AB}, \mathrm{C}, \mathrm{D}, \mathrm{E}$, and F), we generated two clusters, with and without witnesses, using Tocher's optimization method (Cruz et al., 2012).

\section{Joint analysis of experiments}

The joint analysis of experiments was performed using two methods. The first one was the sum of dissimilarity matrices of each strategy in both experiments, resulting in matrices: $\mathrm{AB}_{1}+\mathrm{AB}_{2}=\mathrm{AB}_{12} ; \mathrm{C}_{1}+\mathrm{C}_{2}=\mathrm{C}_{12} ; \mathrm{D}_{1}+\mathrm{D}_{2}=\mathrm{D}_{12} ; \mathrm{AB}_{12}+$ $D_{12}=E_{12} ; C_{12}+D_{12}=F_{12}$ (Figure 2).

The second joint analysis of experiments was performed by directly integrating the data from both experiments. First for quantitative traits, the overall average was obtained for the accessions considering all individuals in both experiments. After that, genetic distances were obtained using SED, resulting in dissimilarity matrix $\mathrm{G}$ (Strategy 6).

For qualitative data, dissimilarity matrix $\mathrm{H}$ was obtained using WED based on scores of all individuals analyzed (Strategy 7). Finally, the integration of qualitative and quantitative data was obtained by the sum of matrices $\mathrm{G}$ and $\mathrm{H}$, generating dissimilarity matrix I (Strategy 8). In the joint analyses, clusters were also formed, with and without witnesses, using Tocher's optimization method (Figure 2).

A total of 36 clusters were obtained through the eight strategies. To facilitate the interpretation of these results, the dissimilarity between different clusters was calculated using the analysis of multicategorical descriptors with binary pattern proposed by Cole-Rodgers et al. (1997):

$$
d_{i i^{\prime}}=\frac{1}{v} \sum_{j=1}^{v} \frac{b_{j}+c_{j}}{a_{j}+b_{j}+c_{j}+d_{j}}
$$

Where: $a_{j}$ and $d_{j}$ are the number of agreements types 1-1 and $0-0$ for the $\mathrm{j}^{\text {th }}$ variable, respectively; $b_{j}$ and $c_{j}$ are the number of disagreements types 1-0 and $0-1$ for the $j^{\text {th }}$ variables, respectively. 
Each cluster represented a treatment and each group represented a multicategorical variable with 22 or 20 classes for treatments with or without witnesses, respectively. Hence, when an accession belonged to a group, it was scored 1, and if it were absent, it was scored 0. After data tabulation, we performed dissimilarity and cluster analyses using Tocher's method for clusters of each experiment separately and for those derived from the joint analysis.

\section{Conclusion}

The sum of matrices obtained by the Average Standardized Euclidean Distance and Weighted Average Euclidean Distance is the most suitable strategy for the integration of quantitative and qualitative descriptors of watermelon accessions, respectively. The matrices obtained through this strategy in different assays must be summed up, so that one single matrix is obtained for studies of genetic diversity in watermelon germplasm.

\section{Acknowledgments}

The authors thank the technical and administrative staff of the State University of Bahia. The first author thank the Foundation for Research Support of the State of Bahia for granting scholarship.

\section{References}

Bento CS, Sudré CP, Rodrigues R, Riva EM, Pereira MG (2007) Descritores qualitativos e multicategóricos na estimativa da variabilidade fenotípica entre acessos de pimentas. Scientia Agraria. 8(2):149-156.

Buso GLC, Nass LL, Marques ADA, Lopes CA, Buso JÁ (2004) Avaliação de genótipos de melão, visando identificar fontes de resistência a Acidovorax avenae subsp. citrulli. Brasília: Embrapa Recursos Genéticos e Biotecnologia, Comunicado Técnico. 116.

Cole-Rodgers P, Smith DW, Bosland PW (1997) A novel statistical approach to analyze genetic resource evaluations using Capsicum as an example. Crop Sci. 37:1000-1002.

Crossa J, Franco J (2004) Statistical methods for classifying genotypes. Euphytica. 137 (1):19-37.

Cruz CD, Ferreira FM, Pessoni LA (2011) Biometria aplicada ao estudo da diversidade genética. Suprema, Visconde do Rio Branco. 620.

Cruz CD, Regazzi AJ, Carneiro PCS (2012) Modelos biométricos aplicados ao melhoramento genético. UFV, Viçosa. 514.

Cruz CD (2013) Genes: a software package for analysis in experimental statistics and quantitative genetics. Acta SciAgron. 35 (3):271-276.

Gonçalves LSA, Rodrigues R, Amaral Júnior AD, Karasawa M, Sudré CP (2008) Comparison of multivariate statistical algorithms to cluster tomato heirloom accessions. Genet Mol Res. 7 (4):1289-1297.

Gower JCA (1971) A general coefficient of similarity and some of its properties. Biometrics. 27:857-871.

Lima Neto IS (2013) Pré-melhoramento de abóbora (Cucurbita moschata Duch.) visando biofortificação em carotenóides. Tese (Doutorado em Fitotecnia) - Universidade Federal de Viçosa, Viçosa, MG.

Marim BG, Silva DJH, Carneiro PCS, Miranda GV, Mattedi AP, Caliman FRB (2009) Variabilidade genética e importância relativa de caracteres em acessos de germoplasma de tomateiro. Pesqui Agropecu Bras. 44:1283-1290.

Martins FA, Carneiro PCS, Silva DJH, Cruz CD, Carneiro JES (2012) Integração de dados em estudos de diversidade genética de tomateiro. Pesqui Agropecu Bras. 46 (11):1496-1502.
Mohammadi AS, Prasanna BM (2003) Analysis of genetic diversity in crop plants: salient statistical tools and considerations. Crop Sci. 43 (4):1235-1248.

Neitzke RS, Barbierri RL, Rodrigues, WF, Corrêa IV, Carvalho FIF (2010) Dissimilaridade genética entre acessos de pimenta com potencial ornamental. Hortic Bras. 28:47-53.

Oliveira RA, Nunes GHDS, Oliveira DA, Guimarães IP (2008) Divergência genética entre acessos de melancia coletados no Rio Grande do Norte. Revista Brasileira de Ciências Agrárias. 3 (3):213-217.

Pavoine S, Vallet J, Dufour AB, Gachet S, Daniel H (2009) On the challenge of treating various types of variables: application for improving the measurement of functional diversity. Oikos. 118 (3):391-402.

Priori D, Barbieri RL, Castro CM, Oliveira AC, Vilella JC, Mistura CC (2012) Caracterização molecular de variedades crioulas de abóboras com marcadores microssatélites. Hortic Bras. 30:499-506.

Ramalho MAP, Ferreira DF, Oliveira AC (2005) Experimentação em genética e melhoramento de plantas, 2 ed. UFLA, Lavras. 322.

Rocha MC, Gonçalves LSA, Corrêa FM, Rodrigues R, Silva SL, Abboud ACDS, Carmo MGFD (2009) Descritores quantitativos na determinação da divergência genética entre acessos de tomateiro do grupo cereja. Cienc Rural. 39 (3):664670.

Rodrigues R, Bento CS, Silva MGM, Sudré CP (2010) Atividades de caracterização e avaliação em bancos de germoplasma. In: Pereira TNS (ed) Germoplasma: conservação, manejo e uso no melhoramento de plantas. UFV, Viçosa. 25.

Santos SS (2015) Diversidade genética entre e dentro de acessos de melão da agricultura tradicional do Estado do Maranhão. Dissertação (Mestrado em Horticultura Irrigada) Universidade do Estado da Bahia, Juazeiro, BA.

Sarkar RK, Rao AR, Wahi SD, Bhat KVA (2011) Comparative performance of clustering procedures for mixture of qualitative and quantitative data-an application to black gram. Plant Genet Resour-C. 9 (4):523-527, 2011.

Silva MAS, Ramos SRR, Queiroz MA (1997) Avaliação preliminar de 24 acessos de melão (Cucumis melo). Trabalho apresentado no $9^{\circ}$ Congresso Brasileiro de Genética, Maceió, Universidade Federal de Alagoas, 1997.

Silva ML, Queiroz MA, Ferreira MAJF, Buso GSC (2006) Caracterização morfológica e molecular de acessos de melancia. Hortic Bras. 24:405-409.

Silva ML, Queiroz MA, Ferreira MAJDF, Aragão CA (2007) Variabilidade genética de acessos de melancia coletados em três regiões do estado da Bahia. Rev Caatinga. 20 (4):93-100.

Souza FF, Queiroz MA (2004) Avaliação de caracteres morfológicos úteis na identificação de plantas poliplóides de melancia. Hortic Bras. 22 (3):516-520.

Sudré CP, Cruz CD, Rodrigues R, Riva EM, Amaral Júnior AT, Silva DJH, Pereira TNS (2006) Variáveis multicategóricas na determinação da divergência genética entre acessos de pimenta e pimentão. Hortic Bras. 24 (1):88-93.

Sudré CP, Leonardedecz E, Rodrigues R, Amaral Júnior AT, Moura MDC, Gonçalves, LS (2007) Genetic resources of vegetable crops: a survey in the Brazilian germplasm collections pictured through papers published in the journals of the Brazilian Society for Horticultural Science. Hortic Bras. 25 (4):496-503.

Syafii M, Cartika I, Ruswandi D (2015) Multivariate Analysis of Genetic Diversity among some Maize Genotypes under MaizeAlbizia Cropping System in Indonesia. Asian Journal of Crop Science 7 (4):244-255.

Torres FE, Valle CBD, Lempp B, Teodoro PE, Rigon JPG, Ribeiro LP, Corrêa CCG, Luz Júnior RAA (2015) Estimativa da divergência entre ecótipos de braquiária baseada em descritores quantitativos e qualitativos. Cienc Rural. 45 (3):485-491. 